\title{
Vibration Analysis of Conical Shells by the Improved Fourier Expansion-Based Differential Quadrature Method
}

\author{
Wanyou Li, Gang Wang, and Jingtao Du \\ College of Power and Energy Engineering, Harbin Engineering University, Harbin 150001, China \\ Correspondence should be addressed to Wanyou Li; hrbeu_ripet_lwy@163.com
}

Received 10 August 2015; Accepted 13 September 2015

Academic Editor: Laurent Mevel

Copyright (C) 2016 Wanyou Li et al. This is an open access article distributed under the Creative Commons Attribution License, which permits unrestricted use, distribution, and reproduction in any medium, provided the original work is properly cited.

\begin{abstract}
An improved Fourier expansion-based differential quadrature (DQ) algorithm is proposed to study the free vibration behavior of truncated conical shells with different boundary conditions. The original function is expressed as the Fourier cosine series combined with close-form auxiliary functions. Those auxiliary functions are introduced to ensure and accelerate the convergence of series expansion. The grid points are uniformly distributed along the space. The weighting coefficients in the DQ method are easily obtained by the inverse of the coefficient matrix. The derivatives in both the governing equations and the boundaries are discretized by the DQ method. Natural frequencies and modal shapes can be easily obtained by solving the numerical eigenvalue equations. The accuracy and stability of this proposed method are validated against the results in the literature and a very good agreement is observed. The centrosymmetric properties of these newly proposed weighting coefficients are also validated. Studies on the effects of semivertex angle and the ratio of length to radius are reported.
\end{abstract}

\section{Introduction}

Conical shells are widely used in various engineering fields, such as aerospace and ship industries. The development of accurate shell theories has been the subject of significant research interest for many years, and a large number of shell theories based on different approximations and assumptions have been proposed. However, more work is focused on the vibration of cylindrical shells compared with the conical shell. Since the conical coordinate system is function of the meridional direction, the equations of motion for conical shells consist of a set of partial differential equations with variable coefficients. Current methods for the free vibration analysis of thin conical shells can be classified as to analytical methods and numerical methods. Saunders et al. [1], Garnet and Kempner [2], Siu [3], and Lim and Liew [4] have studied the free vibration of uniform conical shells by Rayleigh-Ritz method. Ueda [5] analyzed the same problems using the finite element method. Irie et al. [6] studied a conical shell with variable thickness by the transfer matrix method. The DQ method was employed to analyze the free vibration of a uniform conical shell [7]. Jin et al. [8] studied the free and forced vibration of conical shell using the improved Fourier series method by considering the general boundary conditions. The kernel particle $(k-p)$ functions were employed in hybridized form with harmonic functions to study the vibration of the conical shell based on Ritz method [9].

Besides the studies of the isotropic conical shells, laminated and functional graded conical shells have also been fully studied by various methods [10-16]. The differential quadrature $(D Q)$ method was adopted to solve the differential governing equations of the conical shell in those researches [10-13]. For the vibration of the rotating conical shell, in which the centrifuge force should be taken into consideration, the DQ method was also extensively used to study those problems $[10,17]$. The reason that the DQ method is widely adopted to study the vibration behavior of the conical shell is the convenience of transforming the partial differential governing equations approximately into a set of linear algebraic governing equations. Imposing the given boundary conditions, the numerical eigenvalue equations for the free vibration of the (rotating or composite) conical shell are derived and solved.

The differential quadrature method is a numerical technique for solving the differential equations. It was first developed by Bellman et al. $[18,19]$ and their associates in 
the early 1970s. The DQ method, akin to the conventional integral quadrature method, approximates the derivative of a function at any location by a linear summation of all the functional values along a mesh line. The key procedure in the DQ method applications lies in the determination of the weighting coefficients. Shu $[7,12,20,21]$ proposed two types of weighting coefficients obtained by the polynomials and the truncated Fourier series, among which the Lagrange interpolation functions are widely used for their simplicity explicitness [7, 21]. Although it is well known that Lagrange interpolation functions are limited by the number of interpolation points and severe oscillation may take place if the order is large, the use of the Gauss-Chebyshev points [7, 21] can accelerate the convergence rate of the DQ method. Some works focusing on improving the accuracy and stability of the DQ method are presented by proposing different ways to generate the weighting coefficients and to determine the distribution of grid points [22-24].

The Fourier series with auxiliary functions was first proposed by Li [25] to study the vibration problems of the beam structure. This method is extensively used to study the $2 \mathrm{D}$ and $3 \mathrm{D}$ structural vibration and vibroacoustic problems [26-30]. The auxiliary functions are introduced to accelerate the convergence and deal with all the possible discontinuities, at the end points or edges, associated with the original Fourier cosine series. This improved Fourier series method becomes a promising method to study the structural vibration problems.

In this paper, the improved Fourier expansion-based differential quadrature method is proposed to solve the free vibration behavior of the truncated conical shell. The weighting coefficients are obtained based on this improved Fourier series in a much easier way. The centrosymmetric properties of these newly proposed weighting coefficients are also validated. The following sections will illustrate the development of this hybrid method, and numerical results are then presented to validate the effectiveness, accuracy, and stability of this current method on predicting the modal characteristics of the conical shell.

\section{Theoretical Formulation}

For a continuous function $f(x)$ defined on $[0, L]$ with an absolutely integrable derivative, it can be expanded in Fourier cosine series:

$$
f(x)=\sum_{m=0}^{\infty} a_{m} \cos \frac{m \pi}{L} x, \quad 0<x<L .
$$

The first-order derivative of $f(x)$ can be done term-byterm:

$$
f^{\prime}(x)=-\sum_{m=0}^{\infty} a_{m} \frac{m \pi}{L} \sin \frac{m \pi}{L} x .
$$

The second-order derivative of $f(x)$ cannot be obtained term-by-term, which is shown as

$$
\begin{aligned}
& f^{\prime \prime}(x)=-\frac{f^{\prime}(L)-f^{\prime}(0)}{L} \\
& -\sum_{m=1}^{\infty}\left(\frac{2}{L}\left[(-1)^{m} f(L)-f(0)\right]+\frac{m \pi}{L} a_{m}\right) \\
& . \frac{m \pi}{L} \cos \frac{m \pi}{L} x .
\end{aligned}
$$

These formulations basically tell that while a cosine series can always be differentiated term-by-term, this can be done to a sine series only if $f^{\prime}(0)=f^{\prime}(L)=0$. To implement the differential quadrature algorithm, the auxiliary functions are added to traditional Fourier cosine series to cover the discontinuity of the function at the end points and to get the derivatives term-by-term. A function can be expanded as [27]

$$
f(x)=\sum_{m=0}^{\infty} a_{m} \cos \frac{m \pi}{L} x+\sum_{t=1}^{4} C_{t} \xi_{t}(x)
$$

where

$$
\begin{aligned}
& \xi_{1}(x)=\frac{9 L}{4 \pi} \sin \left(\frac{\pi x}{2 L}\right)-\frac{L}{12 \pi} \sin \left(\frac{3 \pi x}{2 L}\right) \\
& \xi_{2}(x)=-\frac{9 L}{4 \pi} \cos \left(\frac{\pi x}{2 L}\right)-\frac{L}{12 \pi} \cos \left(\frac{3 \pi x}{2 L}\right) \\
& \xi_{3}(x)=\frac{L^{3}}{\pi^{3}} \sin \left(\frac{\pi x}{2 L}\right)-\frac{L^{3}}{3 \pi^{3}} \sin \left(\frac{3 \pi x}{2 L}\right) \\
& \xi_{4}(x)=-\frac{L^{3}}{\pi^{3}} \cos \left(\frac{\pi x}{2 L}\right)-\frac{L^{3}}{3 \pi^{3}} \cos \left(\frac{3 \pi x}{2 L}\right),
\end{aligned}
$$

where $a_{m}$ and $C_{t}$ represent the unknown Fourier expansion coefficients. The supplementary functions $\xi_{t}(x)$ can be represented as arbitrary continuous functions, regardless of the boundaries. It is easy to verify that $\xi_{1}^{\prime}(0)=\xi_{3}^{\prime \prime \prime}(0)=$ $\xi_{2}^{\prime \prime}(0)=\xi_{4}^{\prime \prime \prime}(0)=1$ and all the other 1st-order and 3rd-order derivatives are identically equal to zero at both ends. The main purpose of introducing these supplementary functions to standard Fourier series is to get the first four derivatives of the Fourier cosine series term-by-term. As an immediate numerical benefit, the Fourier series in (4) will converge uniformly at an accelerated rate.

To implement the differential quadrature method, $N$ points are equally distributed on $[0, L]$ :

$$
x_{i}=\frac{(i-1) L}{N-1}, \quad i=1,2,3, \ldots, N .
$$

The functional values at those grid points can be determined as

$$
f\left(x_{i}\right)=\sum_{m=0}^{\infty} a_{m} \cos \frac{m \pi}{L} x_{i}+\sum_{t=1}^{4} C_{t} \xi_{t}\left(x_{i}\right) .
$$

The Fourier series is truncated to $m=M$. Rewrite (7) into the matrix form 


$$
\left\{\begin{array}{c}
f\left(x_{1}\right) \\
f\left(x_{2}\right) \\
\vdots \\
f\left(x_{N}\right)
\end{array}\right\}=\left[\begin{array}{ccccccccc}
\cos \frac{0 \pi}{L} x_{1} & \cos \frac{1 \pi}{L} x_{1} & \cdots & \cos \frac{M \pi}{L} x_{1} & \xi_{1}\left(x_{1}\right) & \xi_{2}\left(x_{1}\right) & \xi_{3}\left(x_{1}\right) & \xi_{4}\left(x_{1}\right) \\
\cos \frac{0 \pi}{L} x_{2} & \cos \frac{1 \pi}{L} x_{2} & \cdots & \cos \frac{M \pi}{L} x_{2} & \xi_{1}\left(x_{2}\right) & \xi_{2}\left(x_{2}\right) & \xi_{3}\left(x_{2}\right) & \xi_{4}\left(x_{2}\right) \\
\vdots & \vdots & \ddots & \vdots & \vdots & \vdots & \vdots & \vdots \\
\cos \frac{0 \pi}{L} x_{N} & \cos \frac{1 \pi}{L} x_{N} & \cdots & \cos \frac{M \pi}{L} x_{N} & \xi_{1}\left(x_{N}\right) & \xi_{2}\left(x_{N}\right) & \xi_{3}\left(x_{N}\right) & \xi_{4}\left(x_{N}\right)
\end{array}\right]\left\{\begin{array}{c}
a_{0} \\
a_{1} \\
\vdots \\
a_{M} \\
C_{1} \\
C_{2} \\
C_{3} \\
C_{4}
\end{array}\right\} .
$$

The Fourier series coefficients can be obtained by the in which inverse of the matrix

$$
\left\{\begin{array}{c}
a_{0} \\
a_{1} \\
\vdots \\
a_{M} \\
C_{1} \\
C_{2} \\
C_{3} \\
C_{4}
\end{array}\right\}=\mathbf{R}^{-1}\left\{\begin{array}{c}
f\left(x_{1}\right) \\
f\left(x_{2}\right) \\
\vdots \\
f\left(x_{N}\right)
\end{array}\right\},
$$

$$
\mathbf{R}=\left[\begin{array}{cccccccc}
\cos \frac{0 \pi}{L} x_{1} & \cos \frac{1 \pi}{L} x_{1} & \cdots & \cos \frac{M \pi}{L} x_{1} & \xi_{1}\left(x_{1}\right) & \xi_{2}\left(x_{1}\right) & \xi_{3}\left(x_{1}\right) & \xi_{4}\left(x_{1}\right) \\
\cos \frac{0 \pi}{L} x_{2} & \cos \frac{1 \pi}{L} x_{2} & \cdots & \cos \frac{M \pi}{L} x_{2} & \xi_{1}\left(x_{2}\right) & \xi_{2}\left(x_{2}\right) & \xi_{3}\left(x_{2}\right) & \xi_{4}\left(x_{2}\right) \\
\vdots & \vdots & \ddots & \vdots & \vdots & \vdots & \vdots & \vdots \\
\cos \frac{0 \pi}{L} x_{N} & \cos \frac{1 \pi}{L} x_{N} & \cdots & \cos \frac{M \pi}{L} x_{N} & \xi_{1}\left(x_{N}\right) & \xi_{2}\left(x_{N}\right) & \xi_{3}\left(x_{N}\right) & \xi_{4}\left(x_{N}\right)
\end{array}\right] .
$$

In this proposed method, the number of truncated Fourier series and the number of grid points follow the relation that $N=M+5$ to ensure $\mathbf{R}$ is a square matrix to let the inverse be more accurate. Once the constant matrix $\mathbf{R}$ is determined, the approximated Fourier series coefficients are obtained. When the DQ method was first developed, polynomials were adopted to follow this procedure to generate weighting coefficients which would lead to highly ill condition when $N$ is large. The Fourier series, however, show much more stability to derive the coefficients by the inversion of $R$ which will be validated in the results section.

The first-order derivatives at those grid points are

$$
\begin{aligned}
f^{\prime}\left(x_{i}\right)=\sum_{m=0}^{M} a_{m}\left(-\frac{m \pi}{L}\right) \sin \frac{m \pi}{L} x_{i}+\sum_{t=1}^{4} C_{t} \xi_{t}^{\prime}\left(x_{i}\right), & \\
i & =1,2,3, \ldots, N .
\end{aligned}
$$

Rewrite (11) into the matrix form,

$$
\left\{\begin{array}{c}
f^{\prime}\left(x_{1}\right) \\
f^{\prime}\left(x_{2}\right) \\
\vdots \\
f^{\prime}\left(x_{N}\right)
\end{array}\right\}=\mathbf{R}^{(1)} *\left\{\begin{array}{c}
a_{0} \\
a_{1} \\
\vdots \\
a_{M} \\
C_{1} \\
C_{2} \\
C_{3} \\
C_{4}
\end{array}\right\}
$$




$$
\begin{gathered}
=\mathbf{R}^{(1)} * \mathbf{R}^{-1} *\left\{\begin{array}{c}
f\left(x_{1}\right) \\
f\left(x_{2}\right) \\
\vdots \\
f\left(x_{N}\right)
\end{array}\right\} \\
=\mathbf{c}^{(1)} *\left\{\begin{array}{c}
f\left(x_{1}\right) \\
f\left(x_{2}\right) \\
\vdots \\
f\left(x_{N}\right)
\end{array}\right\},
\end{gathered}
$$

in which $\mathbf{R}^{(1)}$ is the first-order derivative of $\mathbf{R}$ and $\mathbf{c}^{(1)}$ is the first-order weighting coefficient matrix of the DQ method: $\mathbf{c}^{(1)}=\mathbf{R}^{(1)} * \mathbf{R}^{-1}$. It is obvious from the above equation that the weighting coefficients of the second- and higher-order derivatives can be completely determined through the same way, which are expressed as

$$
\begin{gathered}
\left\{\begin{array}{c}
f^{(i)}\left(x_{1}\right) \\
f^{(i)}\left(x_{2}\right) \\
\vdots \\
f^{(i)}\left(x_{N}\right)
\end{array}\right\}=\mathbf{R}^{(i)} * \mathbf{R}^{-1} *\left\{\begin{array}{c}
f\left(x_{1}\right) \\
f\left(x_{2}\right) \\
\vdots \\
f\left(x_{N}\right)
\end{array}\right\} \\
=\mathbf{c}^{(i)} *\left\{\begin{array}{c}
f\left(x_{1}\right) \\
f\left(x_{2}\right) \\
\vdots \\
f\left(x_{N}\right)
\end{array}\right\},
\end{gathered}
$$

in which $\mathbf{R}^{(i)}(i=1,2,3,4)$ is the $i$ th-order derivative of $\mathbf{R}$ and $\mathbf{c}^{(i)}$ is the $i$ th-order weighting coefficient matrix of the DQ method: $\mathbf{c}^{(i)}=\mathbf{R}^{(i)} * \mathbf{R}^{-1}$.

In this paper, only four supplementary functions are added to the Fourier cosine series, which will ensure the firstfour-order derivatives to converge at a high rate and to keep stability of the Fourier series. Consequently first-four-order weighting coefficient matrixes can be obtained which are sufficiently enough to study the vibration of a conical shell. Adding more supplementary functions to the Fourier cosine series will give the capability to study the corresponding higher-order partial differential equations.

\section{Free Vibration Behavior of a Conical Shell}

The free vibration behavior of conical shells has been studied by Shu [7] by the DQ method. This model is adopted again to validate the efficiency, accuracy, and stability of this proposed method.

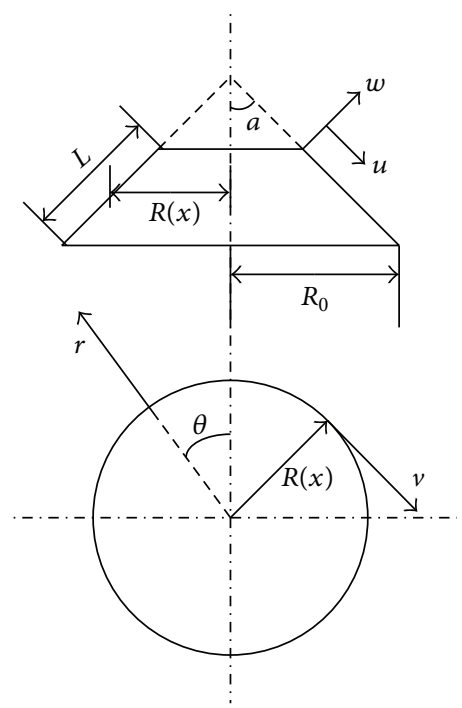

FIGURE 1: Geometry of a conical shell and the coordinate system.

Consider a conical shell structure with semivertex angle $a$ and the radius of the large edge is $R_{0}$, as shown in Figure 1 . The displacement fields of the conical shell in $x, \theta$, and $r$ directions are denoted by $u, v$, and $w$, respectively. If the couplings between these three displacement components are ignored, the field functions can be expressed as

$$
\begin{aligned}
u & =U(x) \cdot \cos (n \theta) \cos (\omega t), \\
v & =V(x) \cdot \sin (n \theta) \cos (\omega t), \\
w & =W(x) \cdot \cos (n \theta) \cos (\omega t),
\end{aligned}
$$

in which $n$ and $\omega$ are the circumferential wave number and the frequency in $\mathrm{rad} / \mathrm{sec}$, respectively.

The differential governing equations of the conical shell based upon Flügge theory are written as

$$
\begin{aligned}
& L_{11} u+L_{12} v+L_{13} w=-\rho h \omega^{2} U, \\
& L_{21} u+L_{22} v+L_{23} w=-\rho h \omega^{2} V, \\
& L_{31} u+L_{32} v+L_{33} w=-\rho h \omega^{2} W,
\end{aligned}
$$

in which $\rho$ and $h$ denote the density of the shell and the shell thickness, respectively. The differential operators $L_{i j}(i, j=$ $1,2,3)$ can be referred to [7]. 
Substituting (14) into (15) and applying (13), then

$$
\begin{aligned}
S_{110} & U_{i}+\sum_{k=1}^{N}\left(S_{111} c_{i k}^{(1)}+S_{112} c_{i k}^{(2)}\right) U_{k}+S_{120} V_{i} \\
& +\sum_{k=1}^{N} S_{121} c_{i k}^{(1)} V_{k}+S_{130} W_{i}+\sum_{k=1}^{N} S_{131} c_{i k}^{(1)} W_{k} \\
& =-\rho h \omega^{2} U_{i} \\
S_{210} & U_{i}+\sum_{k=1}^{N} S_{211} c_{i k}^{(1)} U_{k}+S_{220} V_{i} \\
& +\sum_{k=1}^{N}\left(S_{221} c_{i k}^{(1)}+S_{222} c_{i k}^{(2)}\right) V_{k}+S_{230} W_{i} \\
& +\sum_{k=1}^{N}\left(S_{231} c_{i k}^{(1)}+S_{232} c_{i k}^{(2)}\right) W_{k}=-\rho h \omega^{2} V_{i} \\
& +\sum_{k=1}^{N}\left(S_{331} c_{i k}^{(1)}+S_{232} c_{i k}^{(2)}+S_{333} c_{i k}^{(3)}+S_{334} c_{i k}^{(4)}\right) W_{k} \\
& +\sum_{k=1}^{N}\left(S_{321} c_{i k}^{(1)}+S_{322} c_{i k}^{(2)}\right) V_{k}+S_{330} W_{i} \\
& U_{i}+\sum_{k=1}^{N} S_{311} c_{i k}^{(1)} U_{k}+S_{320} V_{i} \\
&
\end{aligned}
$$

where $c_{i j}^{(n)}$ represents the weighting coefficients, $i=$ $3,4, \ldots, N-2$. follows:

The boundary conditions for the conical shell are as simple-supported boundary condition (S):

$$
\begin{aligned}
V & =0, \\
W & =0, \\
N_{x} & =0, \\
M_{x} & =0,
\end{aligned}
$$

clamped boundary condition (C):

$$
\begin{aligned}
V & =0, \\
W & =0, \\
U & =0, \\
W^{(1)} & =0 .
\end{aligned}
$$

The boundary conditions can also be expressed in the differential forms by substituting (13) into them. Simple supported condition at the small edge is chosen as an example:

$$
\begin{aligned}
& V_{1}=0 \\
& W_{1}=0 \\
& \left(\frac{v \sin a}{R_{1}}+c_{11}^{(1)}\right) U_{1}+c_{1 N}^{(1)} U_{N}=-\sum_{k=2}^{N-1} c_{1 k}^{(1)} U_{k} \\
& \left(\frac{v \sin a}{R_{1}} c_{12}^{(1)}+c_{12}^{(2)}\right) W_{2} \\
& \quad+\left(\frac{v \sin a}{R_{1}} c_{1(N-1)}^{(1)}+c_{1(N-1)}^{(2)}\right) W_{N-1} \\
& \quad=-\sum_{k=3}^{N-2}\left(c_{1 k}^{(2)}+\frac{v \sin a}{R_{2}} c_{1 k}^{(1)}\right) W_{k} .
\end{aligned}
$$

Other boundary conditions can also be formulated in similar forms. Those four boundary formulations are applied at the grid points of $i=1,2, N-2, N$. Rewrite (16a), (16b), (16c), (19a), (19b), (19c), (19d), and boundary conditions into the matrix form

$$
\mathbf{P X}=\Omega \mathbf{X}
$$

where $\Omega=\rho h \omega^{2}, \mathbf{P}$ is a matrix with the dimension of $3 N \times 3 N$, and

$$
\begin{gathered}
\mathbf{X}=\left\{U_{1}, U_{2}, \ldots, U_{N}, V_{1}, V_{2}, \ldots, V_{N}, W_{1}, W_{2}, \ldots,\right. \\
\left.W_{N}\right\}^{T} .
\end{gathered}
$$

It is clear from (20) that the natural frequencies and modal shapes for the conical shell can now be directly obtained by solving the standard matrix eigenvalue problem. The natural frequency parameter is defined as

$$
\Omega_{i}=R_{0} \omega_{i} \sqrt{\frac{\rho\left(1-v^{2}\right)}{E}} .
$$

\section{Numerical Results and Discussion}

4.1. Convergence Study. To study the convergence of this proposed method, different numbers of grid points or truncated Fourier series $(N=M+5)$ are selected. The geometric and material parameters of the conical shell are Young's modulus $E=70 \mathrm{GPa}$, Poisson's ratio $v=0.3, \rho=2700 \mathrm{~kg} / \mathrm{m}^{3}$, $h / R_{0}=0.01, a=45^{\circ}$, and $L \sin a / R_{0}=0.5$. The effect of the number of grid points that affects the natural frequencies is studied. The results derived by finite element method (FEM) are adopted to compare with those obtained by this method. Figure 2 shows the natural frequency parameters under S-S boundary conditions when the circumferential wave number is $n=0$ and axial number is $m=1,2,3$. Figure 3 shows the natural frequency parameter under S-C boundary condition when the circumferential wave number is $n=3$ and axial number is $m=1,2,3$. By comparing the results derived 
TABLE 1: Comparison of the natural frequency parameters under variable circumferential wave numbers $\left(a=45^{\circ}, L \sin a / R_{2}=0.5\right.$, and $\left.h / R_{0}=0.01\right)$.

\begin{tabular}{|c|c|c|c|c|c|c|c|c|c|c|}
\hline \multirow{2}{*}{$n$} & \multicolumn{3}{|c|}{$\mathrm{C}-\mathrm{C}$} & \multicolumn{3}{|c|}{ S-S } & \multicolumn{2}{|c|}{ F-C } & \multicolumn{2}{|c|}{ F-S } \\
\hline & Present & Shu [7] & Irie et al. [6] & Present & Shu [7] & Irie et al. [6] & Present & Irie et al. [6] & Present & Irie et al. [6] \\
\hline 0 & 0.8731 & 0.8732 & 0.8731 & 0.2234 & 0.2233 & 0.2233 & 0.8696 & 0.8696 & 0.1435 & 0.1441 \\
\hline 1 & 0.8120 & 0.8120 & 0.8120 & 0.5460 & 0.5463 & 0.5462 & 0.7634 & 0.7634 & 0.1660 & 0.1667 \\
\hline 2 & 0.6696 & 0.6696 & 0.6696 & 0.6307 & 0.6310 & 0.6310 & 0.5289 & 0.5292 & 0.1152 & 0.1158 \\
\hline 3 & 0.5430 & 0.5428 & 0.5430 & 0.5063 & 0.5062 & 0.5065 & 0.363 & 0.3637 & 0.1007 & 0.1017 \\
\hline 4 & 0.4568 & 0.4566 & 0.4570 & 0.3944 & 0.3942 & 0.3947 & 0.2818 & 0.2829 & 0.1467 & 0.1474 \\
\hline 5 & 0.4092 & 0.4089 & 0.4095 & 0.3341 & 0.3340 & 0.3348 & 0.2767 & 0.2779 & 0.2089 & 0.2093 \\
\hline 6 & 0.3965 & 0.3963 & 0.3970 & 0.3239 & 0.3239 & 0.3248 & 0.3184 & 0.3196 & 0.2729 & 0.2743 \\
\hline 7 & 0.4144 & 0.4143 & 0.4151 & 0.3512 & 0.3514 & 0.3524 & 0.3762 & 0.3775 & 0.3345 & 0.3361 \\
\hline 8 & 0.4569 & 0.4568 & 0.4577 & 0.4019 & 0.4023 & 0.4033 & 0.4398 & 0.4411 & 0.3970 & 0.3985 \\
\hline 9 & 0.5177 & 0.5177 & 0.5186 & 0.4670 & 0.4676 & 0.4684 & 0.5103 & 0.5116 & 0.4655 & 0.4670 \\
\hline
\end{tabular}

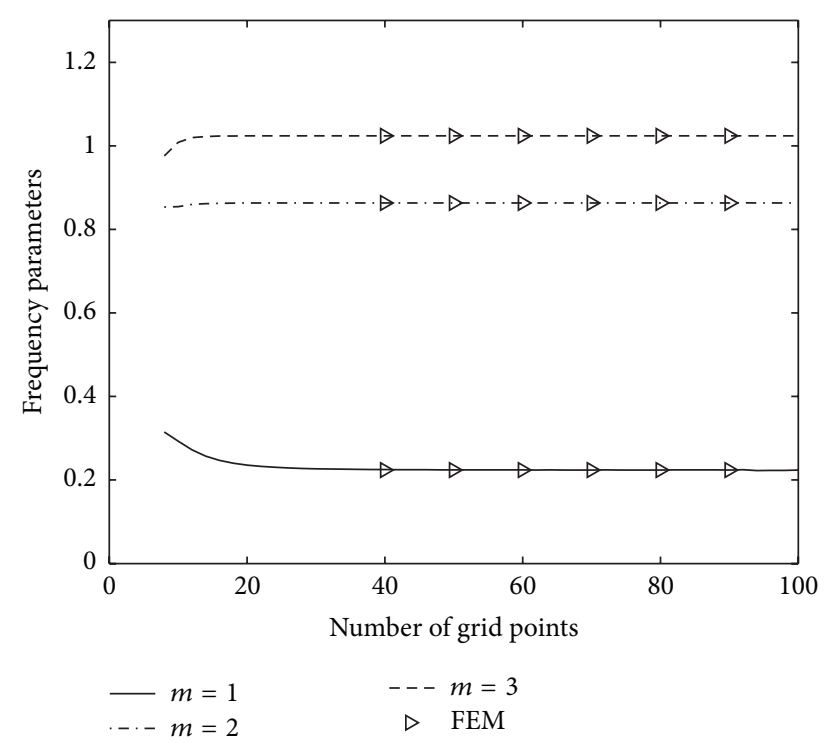

FIGURE 2: Natural frequency parameters under S-S boundary conditions $(n=0, m=1,2,3)$.

by this method and FEM, the fast convergence behavior and high stability of this method are observed. The natural frequency parameters keep stable even with a large number of grid points. In the following calculation, the number of grid points will be chosen as $N=30$.

4.2. Validation of This Proposed Method. To validate the accuracy of the present method, an example reported by Irie et al. [6] and Shu [7] is adopted again. Shu [7] studied the free vibration behavior of this problem by the DQ method, in which Lagrange interpolation functions and Gauss-Chebyshev points are employed. The geometric parameters are $a=45^{\circ}, L \sin a / R_{0}=0.5$, and $h / R_{0}=$ 0.01 . Table 1 shows the comparison between current natural frequency parameters and results studied by Irie et al. [6] and Shu [7] for the conical shells with C-C, S-S, F-C, and F-S boundary conditions. The small discrepancies show a good

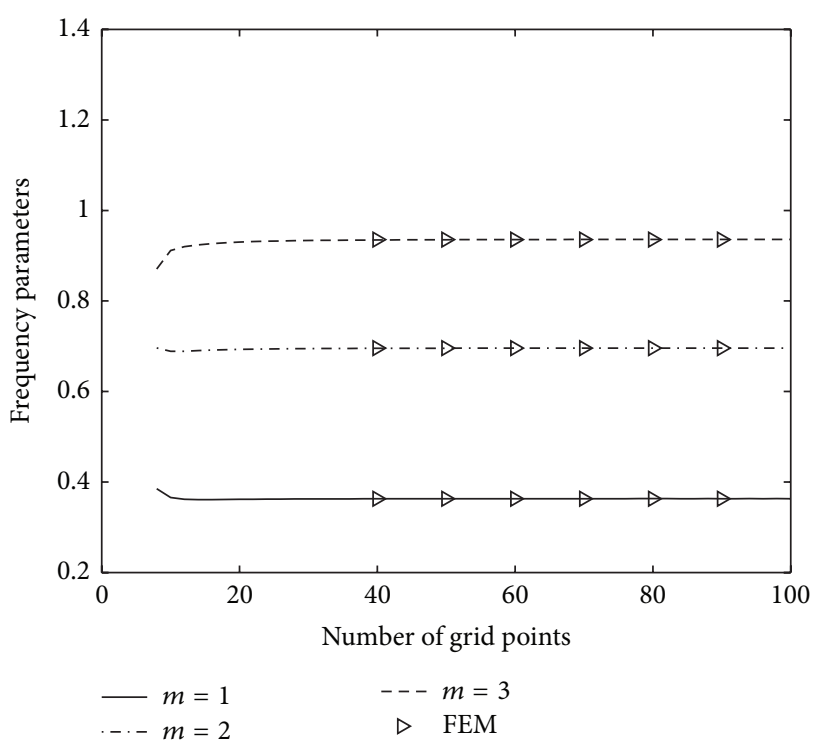

FIgURE 3: Natural frequency parameters under S-C boundary conditions $(n=3, m=1,2,3)$.

agreement. Figures 4 and 5 show some selected modal shapes of the conical shell with different parameters.

4.3. Effects of the Auxiliary Functions. To study the advantage of introducing the auxiliary functions, the weighting coefficients obtained without auxiliary functions are adopted to study the eigenvalue problems. The constant matrix $\mathbf{R}$ without the auxiliary functions can be rewritten as

$$
\mathbf{R}_{\cos }=\left[\begin{array}{cccc}
\cos \frac{0 \pi}{L} x_{1} & \cos \frac{1 \pi}{L} x_{1} & \cdots & \cos \frac{M \pi}{L} x_{1} \\
\cos \frac{0 \pi}{L} x_{2} & \cos \frac{1 \pi}{L} x_{2} & \cdots & \cos \frac{M \pi}{L} x_{2} \\
\vdots & \vdots & \ddots & \vdots \\
\cos \frac{0 \pi}{L} x_{N} & \cos \frac{1 \pi}{L} x_{N} & \cdots & \cos \frac{M \pi}{L} x_{N}
\end{array}\right] .
$$




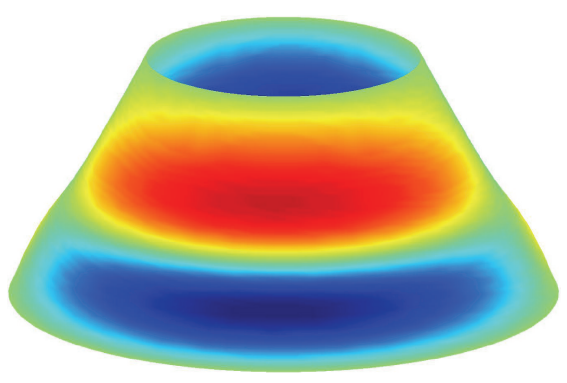

(a) $n=1, m=2$

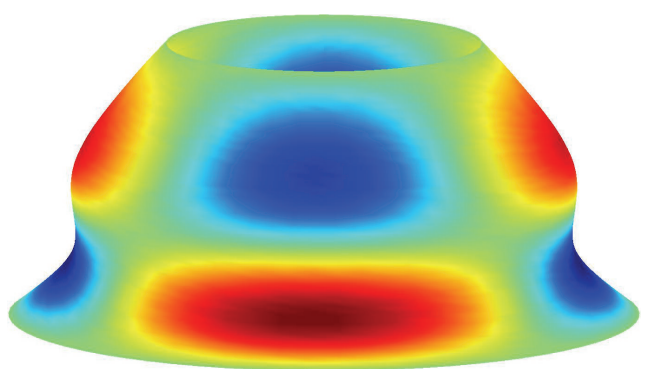

(b) $n=2, m=2$

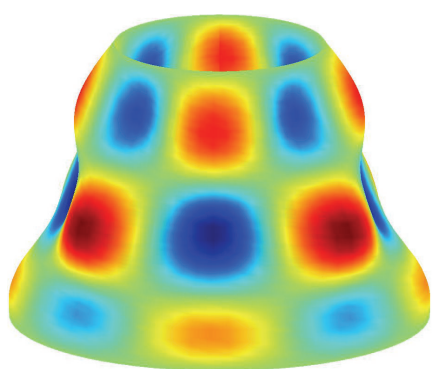

(c) $n=4, m=3$

FIgURE 4: Modal shapes of the conical shell under S-S boundary conditions.

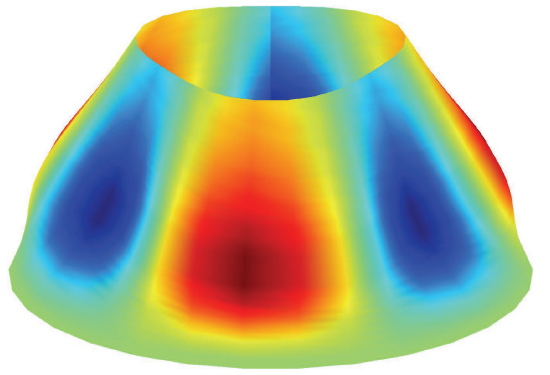

(a) $n=3, m=1$

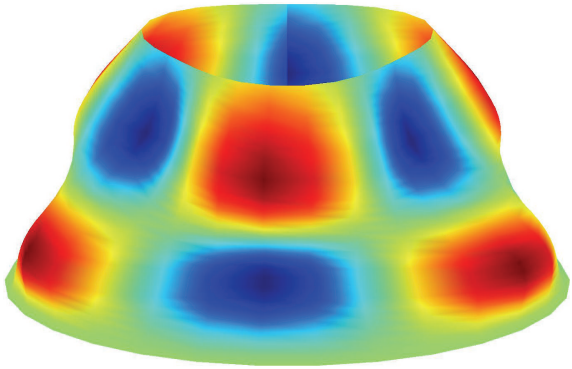

(b) $n=3, m=2$

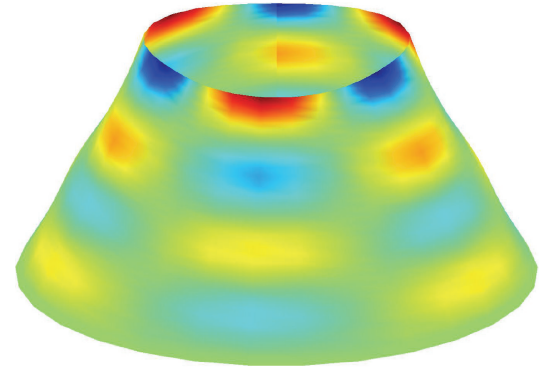

(c) $n=3, m=4$

FIGURE 5: Modal shapes of the conical shell under F-C boundary conditions.

TABLE 2: Natural frequency parameters under different boundary conditions obtained by two types of weighting coefficients $(n=0$, $m=0)$.

\begin{tabular}{lccc}
\hline & F-S & S-S & F-C \\
\hline With auxiliary functions & 0.1435 & 0.2234 & 0.8696 \\
Without auxiliary functions & 0.8442 & 0.8772 & 0.8403 \\
Irie et al. [6] & 0.1441 & 0.2233 & 0.8696 \\
\hline
\end{tabular}

The weighting coefficient matrix is then derived in the same way as (13):

$$
\mathbf{c}_{\mathrm{cos}}^{(i)}=\mathbf{R}_{\mathrm{cos}}^{(i)} * \mathbf{R}_{\mathrm{cos}}^{-1}
$$

in which $\mathbf{c}_{\text {cos }}^{(i)}$ is the $i$ th-order weighting coefficient matrix and $\mathbf{R}_{\text {cos }}^{(i)}$ is the $i$ th-order derivative of $\mathbf{R}_{\text {cos }}$. By using this type of weighting coefficients, natural frequency parameters are derived again to compare with the results obtained before. Table 2 shows the frequency comparison between the results derived by these two types of weighting coefficients. The number of grid points is chosen as $N=35$. It is clear that the introduction of auxiliary functions will improve the accuracy of this method.

4.4. Relation between the Numbers of Truncated Fourier Series and Grid Points. For the study above, the numbers of truncated Fourier series and grid points follow the relation that $N=M+5$ to ensure that $\mathbf{R}$ is a square matrix. It is well known that pseudoinverse of $\mathbf{R}$ could also be adopted to derive weighting coefficients even when $N \neq M+5$. Figure 6 shows the natural frequency parameters when $M$ is set to $M=30$ and $N=10-100$. It is clear that only if $N=35$, that is, $N=M+5$, the accurate results could be obtained. To implement this method, the relation between the numbers of truncated Fourier series and grid points should be strictly set to $N=M+5$.

4.5. The Centrosymmetric Properties of the Weighting Coefficients. When DQ method was developed, the centrosymmetric and skew centrosymmetric properties were observed shown as [21]

$$
\mathbf{c}^{(i)}=\mathbf{c}^{(i-1)} * \mathbf{c}^{(1)} .
$$

Equation (25) shows that the DQ weighting coefficient matrix is skew centrosymmetric for odd derivatives ( $i$ is odd) and centrosymmetric for even order derivatives ( $i$ is even) when the grid distribution is symmetric with respect to the center point. This conclusion is true for both uniform and nonuniform grids.

In this proposed method, the centrosymmetric properties are also validated. Two types of weighting coefficients calculated in different ways are employed to study the eigenvalue problems; those are

$$
\begin{aligned}
& \mathbf{c}^{(i)}=\mathbf{R}^{(i)} * \mathbf{R}^{-1}, \\
& \mathbf{c}^{(i)}=\mathbf{c}^{(i-1)} * \mathbf{c}^{(1)} .
\end{aligned}
$$

To implement (27), $\mathbf{c}^{(1)}$ is first derived based on (26). Table 3 shows the natural frequency parameters obtained by 


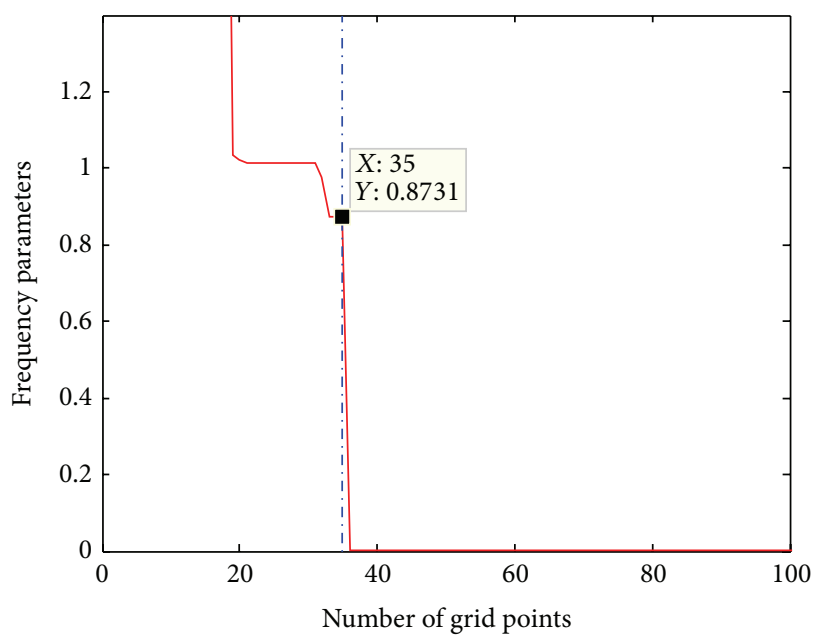

(a) C-C

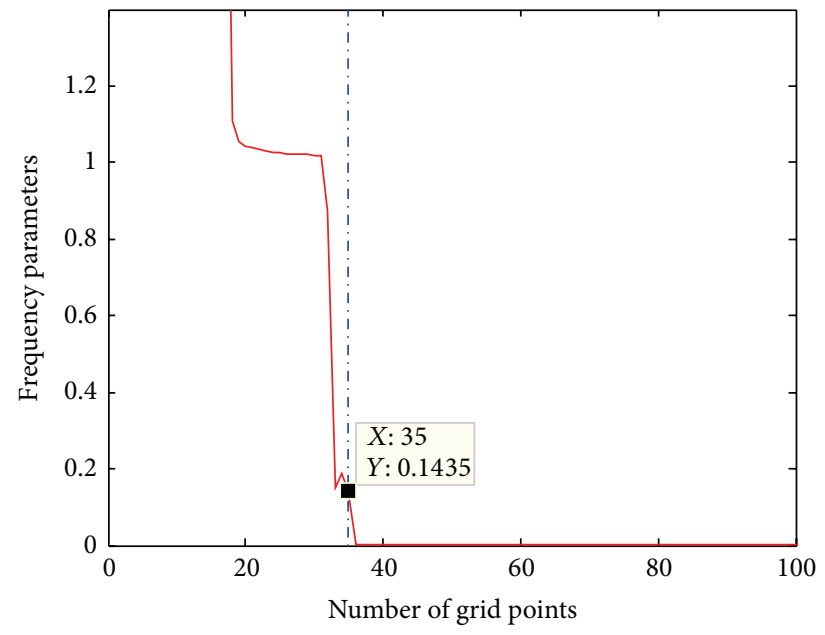

(b) F-S

FIGURE 6: Natural frequency parameters derived by different numbers of grid points when $M=30$ ((a) C-C, (b) F-S).

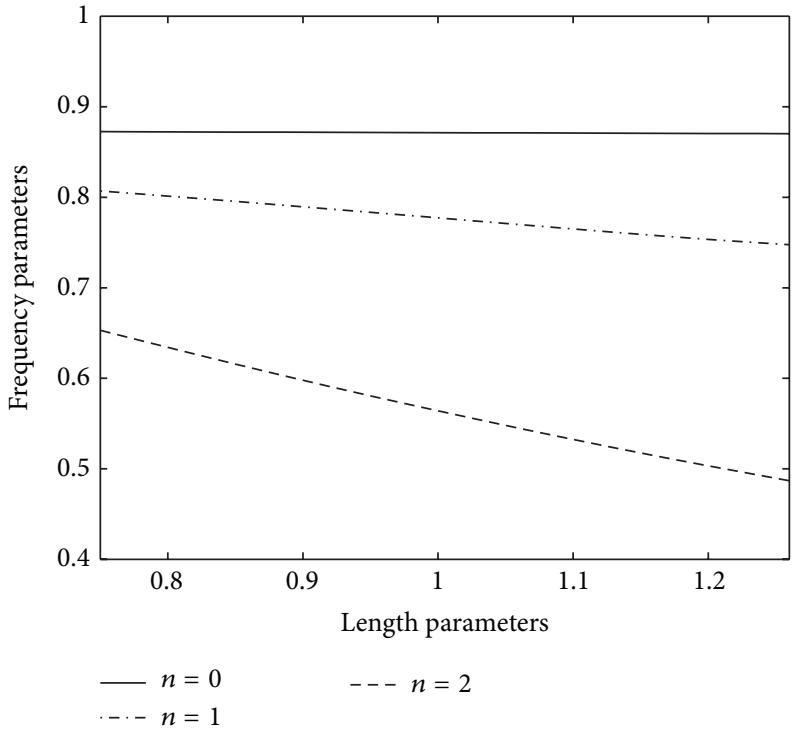

(a)

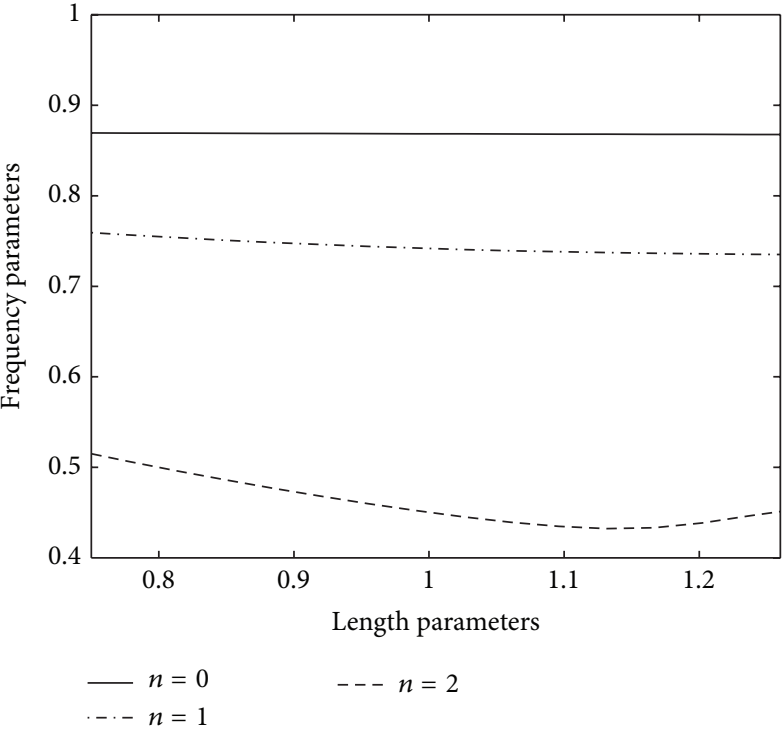

(b)

FIgURE 7: Natural frequency parameters for the conical shell with variation of length to radius $\left(a=45^{\circ}, h / R_{2}=0.01\right.$; (a) C-C, (b) F-C).

these two types of weighting coefficients. The small discrepancies between these two results show a good agreement. It is concluded that the weighting coefficient matrix derived by this proposed method also obeys the centrosymmetric property.

4.6. Effects of Geometric Parameters. The geometric parameters play an important role in affecting the natural frequencies of a conical shell. In this part, two parameters are studied to study their effects on the free vibration behavior of the conical shells. Figure 7 shows the natural frequency parameters changing with variable ratio of length to radius of the conical shell under C-C and F-C boundary conditions. The geometric parameters are chosen as $a=45^{\circ}, h / R_{2}=0.01$, and
TABLE 3: Natural frequency parameters obtained by employing (26) and (27) $(n=0, m=0)$.

\begin{tabular}{lccc}
\hline & F-C & C-C & F-S \\
\hline By employing (27) & 0.8689 & 0.8730 & 0.1440 \\
By employing (26) & 0.8689 & 0.8731 & 0.1435 \\
Irie et al. [6] & 0.8689 & 0.8731 & 0.1441 \\
\hline
\end{tabular}

variable $L \sin a / R_{0}=0.5-0.9$. The frequency parameters nearly keep constant when $n=0$ as increasing the ratio of length to radius. The frequency parameters decrease when $n=1,2$, except for the case of $n=2$ for F-C boundary conditions. Next, the effect of semivertex angle is studied with 


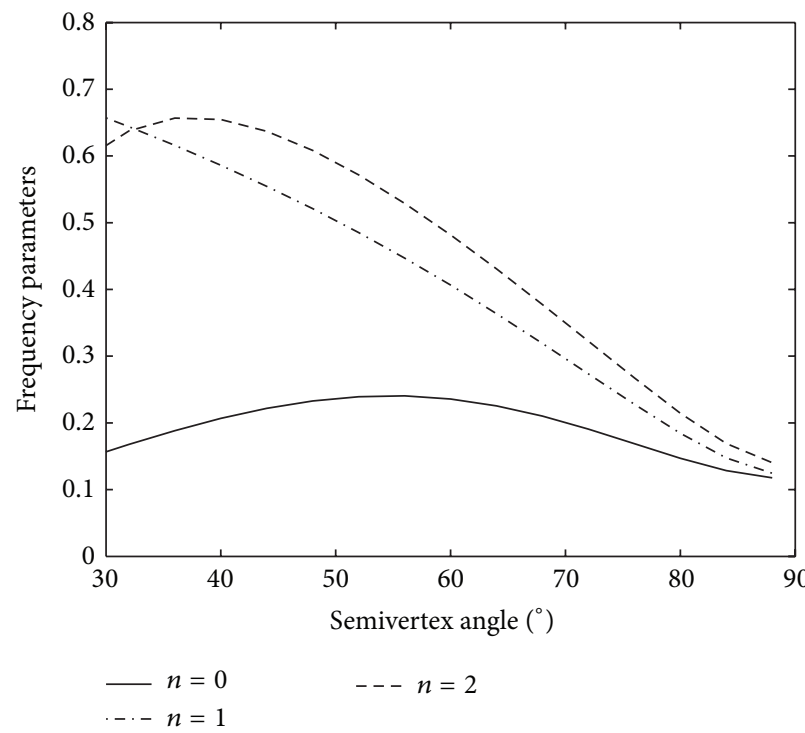

(a)

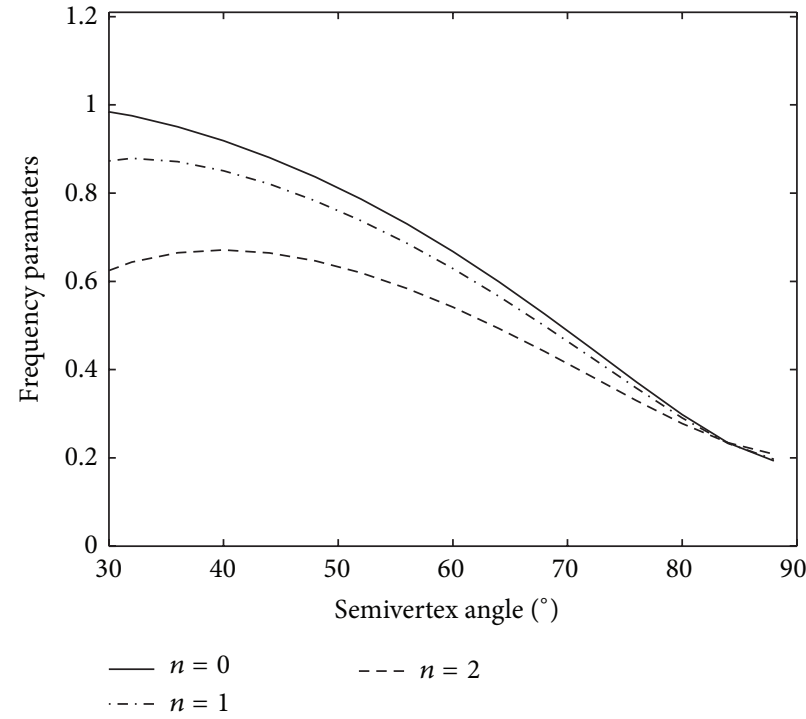

(b)

FIGURE 8: Natural frequency parameters of the conical shell with the variation of semivertex angle $\left(h / R_{2}=0.01, L \sin a / R_{2}=0.5\right.$; (a) S-S, (b) S-C).

the geometric parameters $h / R_{0}=0.01, L \sin a / R_{0}=0.5$, and variable semivertex angle $a=30^{\circ}-90^{\circ}$. Figure 8 shows the natural frequency parameters of different circumferential wave numbers. As semivertex angle increasing to $90^{\circ}$, the frequencies converge to one value. This phenomenon can be explained by the fact that the conical shell degenerates to a circular plate when semivertex angle is $90^{\circ}$.

\section{Conclusions}

In this paper, a new method is proposed to generate the weighting coefficients of the DQ method. The functions in the DQ method are expressed as the Fourier cosine series combined with close-form auxiliary functions. The weighting coefficients are directly derived by the inverse of the constant matrix which presents a much easier way. The boundary conditions and differential governing equations are discretized to form the numerical eigenvalue equations. The results obtained by this method are compared with those available in the literature and a good agreement is observed. The centrosymmetric properties of these newly proposed weighting coefficients are also validated. By increasing the number of grid points, the efficiency and high stability are presented in this method. The effect of those parameters which may affect the dynamic characteristics of the shell is also studied.

This method gives a much easier way to generate weighting coefficients in DQ algorithm. It can also be extended to study higher-order partial differential equations just by adding more corresponding supplementary functions to the Fourier cosine series.

\section{Conflict of Interests}

The authors declare that there is no conflict of interests regarding the publication of this paper.

\section{Acknowledgments}

The research work is supported by the National Natural Science Foundation of China (Grant no. 51375104) and Heilongjiang Province Funds for Distinguished Young Scientists (Grant no. JC 201405).

\section{References}

[1] H. Saunders, E. J. Wisniewski, and P. R. Paslay, "Vibrations of conical shells," The Journal of the Acoustical Society of America, vol. 32, pp. 765-772, 1960.

[2] H. Garnet and J. Kempner, "Axisymmetric free vibrations of conical shells," Journal of Applied Mechanics, vol. 31, no. 3, pp. 458-466, 1966.

[3] C. C. Siu, "Bert, Free vibration analysis of sandwich conical shells with free edges," The Journal of the Acoustical Society of America, vol. 47, no. 3, pp. 943-945, 1970.

[4] C. W. Lim and K. M. Liew, "Vibratory behaviour of shallow conical shells by a global Ritz formulation," Engineering Structures, vol. 17, no. 1, pp. 63-70, 1995.

[5] T. Ueda, "Non-linear free vibrations of conical shells," Journal of Sound and Vibration, vol. 64, no. 1, pp. 85-95, 1979.

[6] T. Irie, G. Yamada, and K. Tanaka, "Natural frequencies of truncated conical shells," Journal of Sound and Vibration, vol. 92, no. 3, pp. 447-453, 1984.

[7] C. Shu, "An efficient approach for free vibration analysis of conical shells," International Journal of Mechanical Sciences, vol. 38, no. 8-9, pp. 935-949, 1996.

[8] G. Jin, X. Ma, S. Shi, T. Ye, and Z. Liu, "A modified Fourier series solution for vibration analysis of truncated conical shells with general boundary conditions," Applied Acoustics, vol. 85, pp. 8296, 2014.

[9] K. M. Liew, T. Y. Ng, and X. Zhao, "Free vibration analysis of conical shells via the element-free kp-Ritz method," Journal of Sound and Vibration, vol. 281, no. 3-5, pp. 627-645, 2005. 
[10] T. Y. Ng, H. Li, and K. Y. Lam, "Generalized differential quadrature for free vibration of rotating composite laminated conical shell with various boundary conditions," International Journal of Mechanical Sciences, vol. 45, no. 3, pp. 567-587, 2003.

[11] F. Tornabene, "Free vibration analysis of functionally graded conical, cylindrical shell and annular plate structures with a four-parameter power-law distribution," Computer Methods in Applied Mechanics and Engineering, vol. 198, no. 37-40, pp. 29112935, 2009.

[12] C. Shu, "Free vibration analysis of composite laminated conical shells by generalized differential quadrature," Journal of Sound and Vibration, vol. 194, no. 4, pp. 587-604, 1996.

[13] C.-P. Wu and C.-Y. Lee, "Differential quadrature solution for the free vibration analysis of laminated conical shells with variable stiffness," International Journal of Mechanical Sciences, vol. 43, no. 8, pp. 1853-1869, 2001.

[14] X. Zhao and K. M. Liew, "Free vibration analysis of functionally graded conical shell panels by a meshless method," Composite Structures, vol. 93, no. 2, pp. 649-664, 2011.

[15] A. Korjakin, R. Rikards, A. Chate, and H. Altenbach, "Analysis of free damped vibrations of laminated composite conical shells," Composite Structures, vol. 41, no. 1, pp. 39-47, 1998.

[16] A. A. Lakis, A. Selmane, and A. Toledano, "Non-linear free vibration analysis of laminated orthotropic cylindrical shells," International Journal of Mechanical Sciences, vol. 40, no. 1, pp. 27-49, 1998.

[17] Ö. Civalek, "An efficient method for free vibration analysis of rotating truncated conical shells," International Journal of Pressure Vessels and Piping, vol. 83, no. 1, pp. 1-12, 2006.

[18] R. Bellman and J. Casti, "Differential quadrature and long-term integration," Journal of Mathematical Analysis and Applications, vol. 34, pp. 235-238, 1971.

[19] R. Bellman, B. G. Kashef, and J. Casti, "Differential quadrature: a technique for the rapid solution of nonlinear partial differential equations," Journal of Computational Physics, vol. 10, pp. 40-52, 1972.

[20] C. Shu, Generalized differential-integral quadrature and application to the simulation of incompressible viscous flows including parallel computation [Ph.D. thesis], University of Glasgow, 1991.

[21] C. Shu, Differential Quadrature and Its Application in Engineering, Springer Science \& Business Media, London, UK, 2000.

[22] Z. Zong, "A variable order approach to improve differential quadrature accuracy in dynamic analysis," Journal of Sound and Vibration, vol. 266, no. 2, pp. 307-323, 2003.

[23] A. G. Striz, X. Wang, and C. W. Bert, "Harmonic differential quadrature method and applications to analysis of structural components," Acta Mechanica, vol. 111, no. 1-2, pp. 85-94, 1995.

[24] H. Zhong, "Spline-based differential quadrature for fourth order differential equations and its application to Kirchhoff plates," Applied Mathematical Modelling, vol. 28, no. 4, pp. 353366, 2004.

[25] W. L. Li, "Free vibrations of beams with general boundary conditions," Journal of Sound and Vibration, vol. 237, no. 4, pp. 709-725, 2000.

[26] W. L. Li, "Vibration analysis of rectangular plates with general elastic boundary supports," Journal of Sound and Vibration, vol. 273, no. 3, pp. 619-635, 2004.

[27] W. L. Li, X. Zhang, J. Du, and Z. Liu, "An exact series solution for the transverse vibration of rectangular plates with general elastic boundary supports," Journal of Sound and Vibration, vol. 321, no. 1-2, pp. 254-269, 2009.
[28] L. Dai, T. Yang, J. Du, W. L. Li, and M. J. Brennan, "An exact series solution for the vibration analysis of cylindrical shells with arbitrary boundary conditions," Applied Acoustics, vol. 74, no. 3, pp. 440-449, 2013.

[29] J. T. Du, W. L. Li, H. A. Xu, and Z. G. Liu, "Vibro-acoustic analysis of a rectangular cavity bounded by a flexible panel with elastically restrained edges," The Journal of the Acoustical Society of America, vol. 131, no. 4, pp. 2799-2810, 2012.

[30] G. Jin, T. Ye, Y. Chen, Z. Su, and Y. Yan, "An exact solution for the free vibration analysis of laminated composite cylindrical shells with general elastic boundary conditions," Composite Structures, vol. 106, pp. 114-127, 2013. 


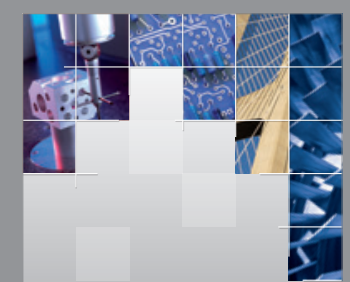

\section{Enfincering}
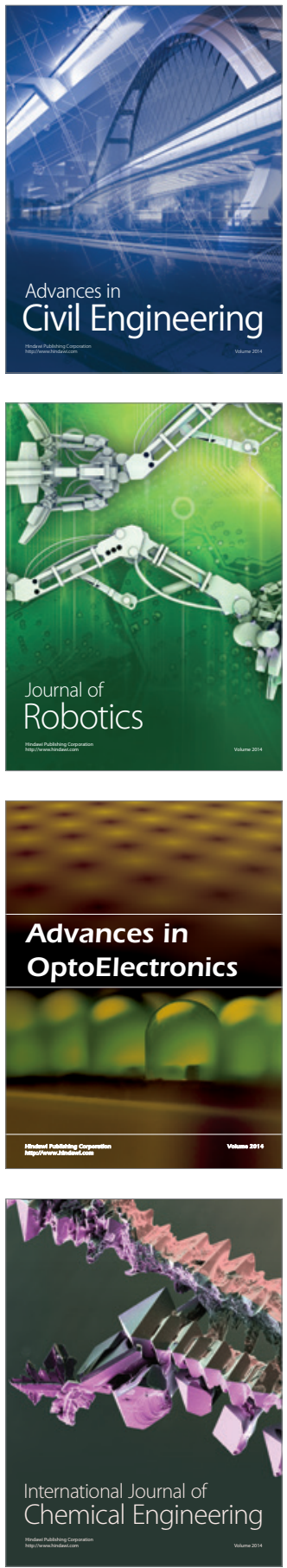

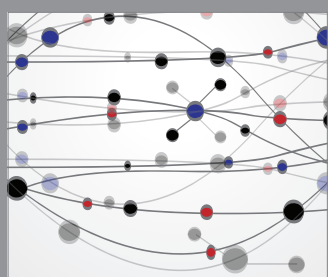

The Scientific World Journal

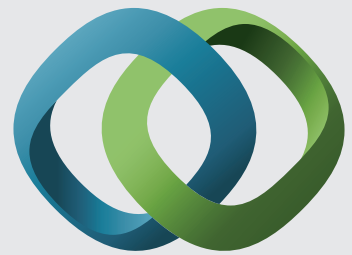

\section{Hindawi}

Submit your manuscripts at

http://www.hindawi.com
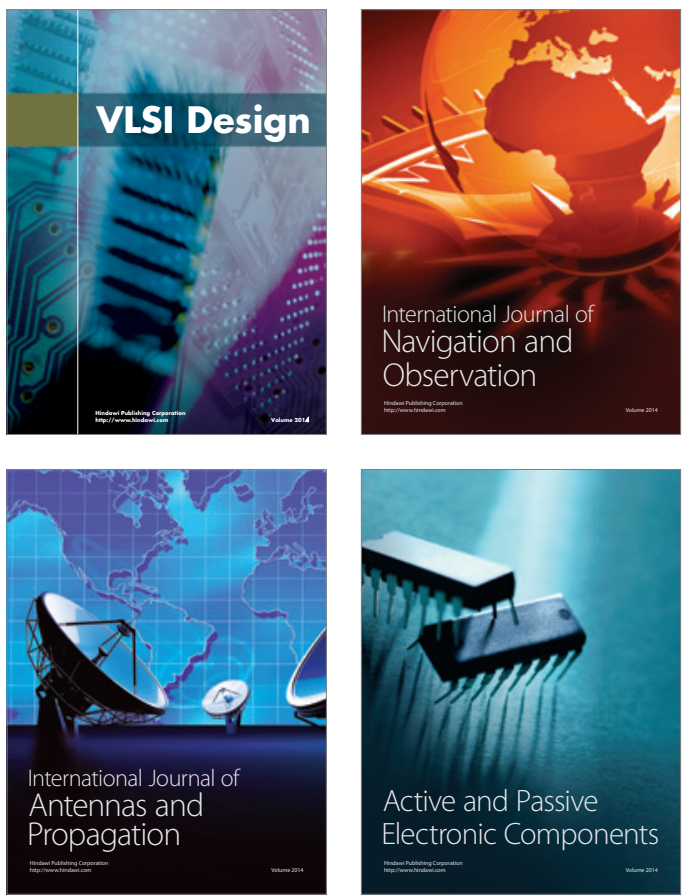
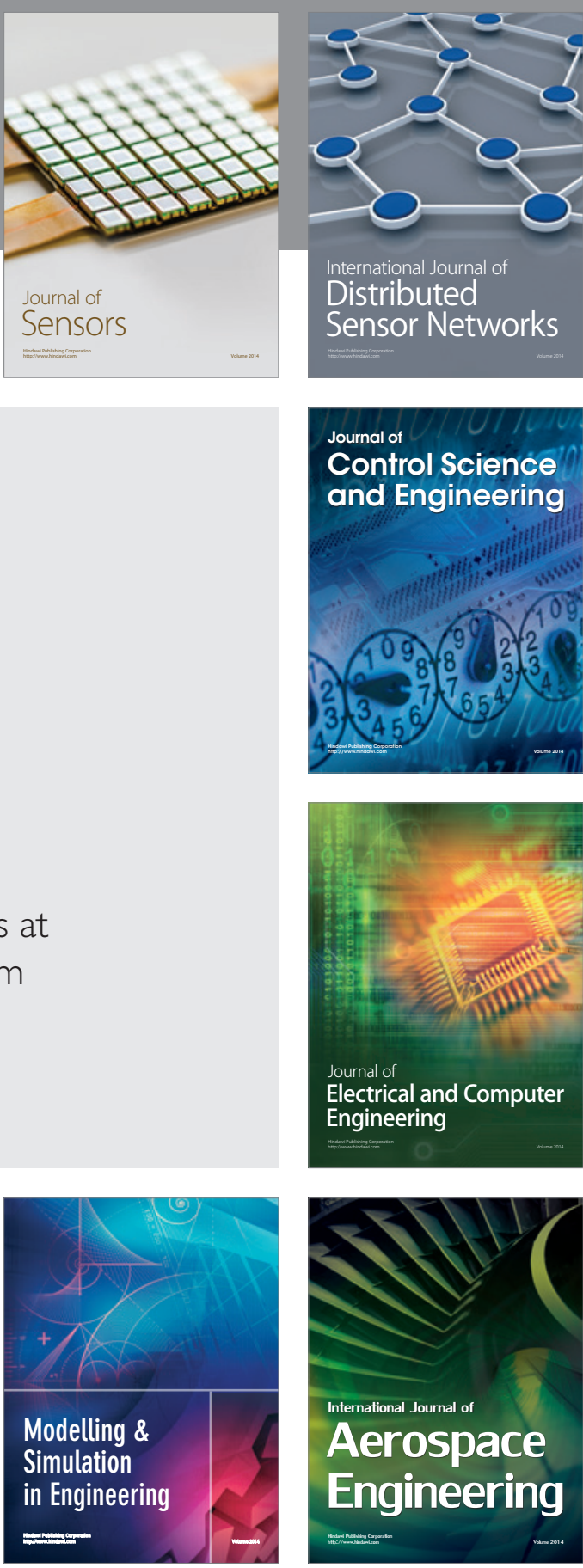

International Journal of

Distributed

Sensor Networks

Journal of

Control Science

and Engineering
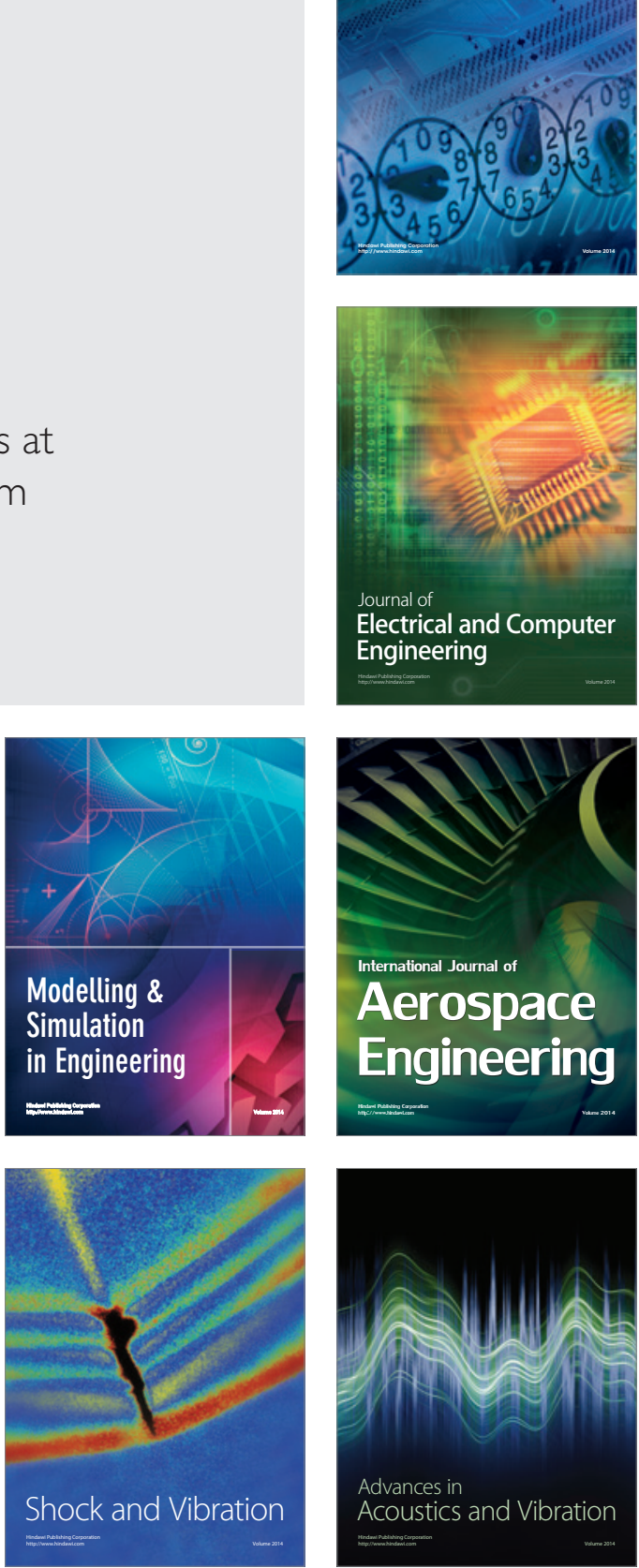